\title{
Erratum: Stress-Induced Changes in Optical Properties, Pigment and Fatty Acid Content of Nannochloropsis sp.: Implications for Non-destructive Assay of Total Fatty Acids
}

\author{
Alexei Solovchenko • Inna Khozin-Goldberg • \\ Lee Recht • Sammy Boussiba
}

Published online: 13 January 2011

(C) Springer Science+Business Media, LLC 2011

Erratum to: Mar Biotechnol

DOI 10.1007/s10126-010-9323-x

The original article was inadvertently published with an incorrect version of Fig. 3. The correct image and caption is shown here:

The online version of the original article can be found at http://dx.doi. org/10.1007/s10126-010-9323-x.

\footnotetext{
A. Solovchenko $(\square)$

Department of Biotechnology, Faculty of Biology,

Moscow State University,

GSP-1 119991, Moscow, Russia

e-mail: wundy@mail.ru

I. Khozin-Goldberg $\cdot$ L. Recht $\cdot$ S. Boussiba

The Microalgal Biotechnology Laboratory,

The Jacob Blaustein Institutes for Desert Research,

Ben-Gurion University of the Negev,

Sede Boker Campus,

Midreshet Ben-Gurion 84990, Israel
} 


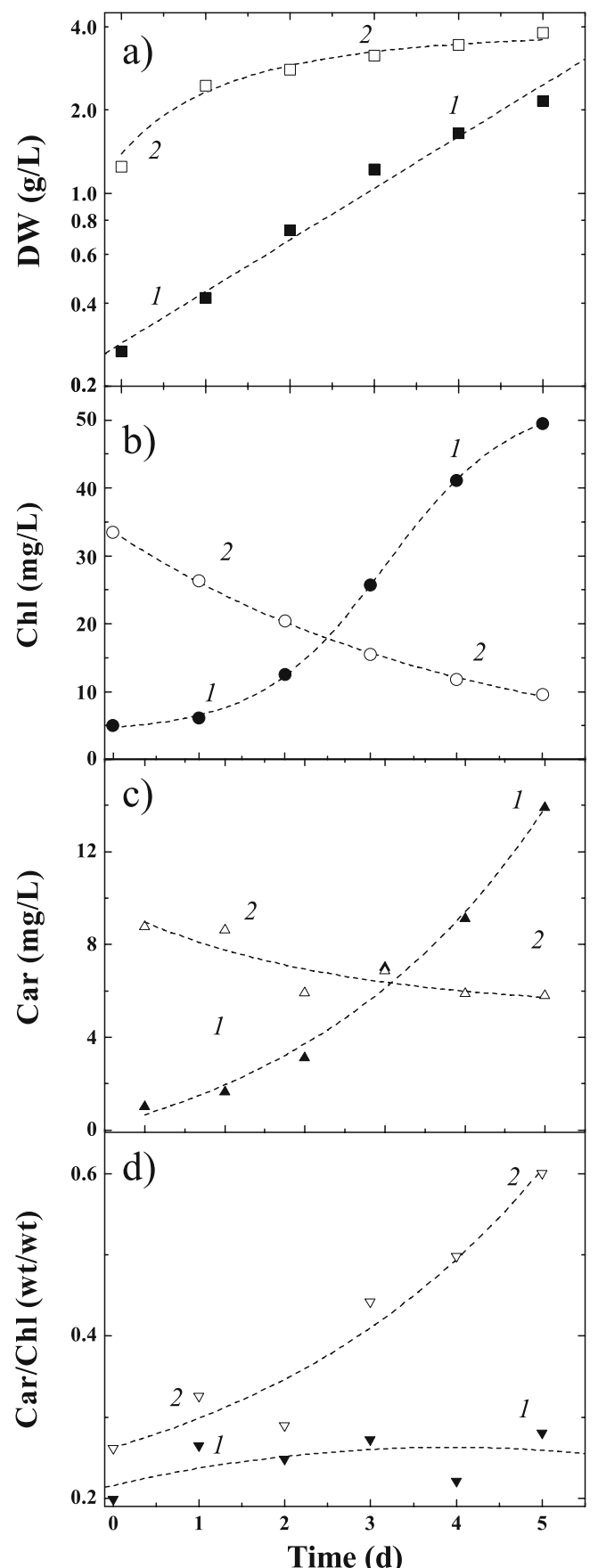

Fig. 3 The time-course of changes in a biomass accumulation, b chlorophyll and $\mathbf{c}$ carotenoid contents and $\mathbf{d}$ their ratio in the Nannochloropsis sp. cultures grown under normal $(75 \mu \mathrm{mol}$ photons $/\left(\mathrm{m}^{2} \mathrm{~s}\right)$ PAR, complete $\mathrm{f} / 2$ medium; curves 1$)$ or stressful (350 $\mu$ mol photons $/\left(\mathrm{m}^{2} \mathrm{~s}\right)$ PAR, N-free $\mathrm{f} / 2$ medium; curves 2) conditions 